\title{
STAT3 links IL-22 signaling in intestinal epithelial cells to mucosal wound healing
}

\author{
Geethanjali Pickert, ${ }^{1}$ Clemens Neufert, ${ }^{2}$ Moritz Leppkes, ${ }^{1}$ Yan Zheng, ${ }^{4}$ \\ Nadine Wittkopf, ${ }^{1}$ Moritz Warntjen, ${ }^{1}$ Hans-Anton Lehr, ${ }^{3}$ Sebastian Hirth, ${ }^{1}$ \\ Benno Weigmann, ${ }^{1}$ Stefan Wirtz, ${ }^{1}$ Wenjun Ouyang, ${ }^{4}$ Markus F. Neurath, ${ }^{5}$ \\ and Christoph Becker ${ }^{1}$
}

IInstitute of Molecular Medicine and 21. Department of Medicine, Johannes Gutenberg University of Mainz, 55131 Mainz, Germany

Institut Universitaire de Pathologie, Centre Hospitalier, Universitaire Vaudois, 1011 Lausanne, Switzerland ${ }^{4}$ Department of Immunology, Genentech, South San Francisco, CA 94080

${ }^{5}$ Department of Medicine 1, University Hospital Erlangen, 91054 Erlangen, Germany

Signal transducer and activator of transcription (STAT) 3 is a pleiotropic transcription factor with important functions in cytokine signaling in a variety of tissues. However, the role of STAT3 in the intestinal epithelium is not well understood. We demonstrate that development of colonic inflammation is associated with the induction of STAT3 activity in intestinal epithelial cells (IECs). Studies in genetically engineered mice showed that epithelial STAT3 activation in dextran sodium sulfate colitis is dependent on interleukin (IL)-22 rather than IL-6. IL-22 was secreted by colonic CD11 $\mathrm{c}^{+}$cells in response to Toll-like receptor stimulation. Conditional knockout mice with an IEC-specific deletion of STAT3 activity were highly susceptible to experimental colitis, indicating that epithelial STAT3 regulates gut homeostasis. STAT3 ${ }^{\text {IEC-KO }}$ mice, upon induction of colitis, showed a striking defect of epithelial restitution. Gene chip analysis indicated that STAT3 regulates the cellular stress response, apoptosis, and pathways associated with wound healing in IECs. Consistently, both IL-22 and epithelial STAT3 were found to be important in wound-healing experiments in vivo. In summary, our data suggest that intestinal epithelial STAT3 activation regulates immune homeostasis in the gut by promoting IL-22-dependent mucosal wound healing.

\section{CORRESPONDENCE}

Christoph Becker:

chbecker@uni-mainz.de

Abbreviations used: DSS, dextran sodium sulfate; IBD, inflammatory bowel disease; IEC, intestinal epithelial cell; LPMC, lamina propria mononuclear cell; pSTAT3, phosphorylated STAT3; TLR, Toll-like receptor; TUNEL, Tdt-mediated dUTP-biotin nick-end labeling.
Inflammatory bowel diseases (IBDs) like Crohn's disease and ulcerative colitis are thought to result from a dysregulated response of the intestinal immune system to bacteria present in the commensal flora (Strober et al., 2007; Rescigno, 2008). To date, it remains unclear whether a breakdown of immune tolerance is the primary cause of these diseases or occurs downstream of an initial defect of the intestinal barrier and intestinal epithelial cells (IECs; Nenci et al., 2007; Strober et al., 2007; Rescigno, 2008).

STAT3 is a transcription factor activated by a variety of cytokines and growth factors (Chapman et al., 2000; Levy and Darnell, 2002). Upon activation, STAT3 translocates to the nucleus, where it regulates genes involved in apoptosis, proliferation, migration, and survival, depending on the cell type (Akira, 2000). STAT3 plays an important role in several autoimmune diseases as well as IBD, where it is ac-

G. Pickert and C. Neufert contributed equally to this paper. tivated in various cell types (Lovato et al., 2003; Mudter et al., 2005). Strikingly, mice with a deletion of STAT3 in hematopoietic cells and particularly in macrophages develop spontaneous colitis, most likely because of the lack of negative regulation of activation (Takeda et al., 1999; Welte et al., 2003; Alonzi et al., 2004). Conversely, mice with a $\mathrm{T}$ cell-specific deletion of STAT3 activity are protected from a variety of autoimmune disease models, highlighting a proinflammatory role of STAT3 in $\mathrm{T}$ cells (Liu et al., 2008). Thus, there is clear evidence for cell type-dependent functions of STAT3 in maintaining intestinal immune homeostasis. In support of this assumption, cytokines like IL-6 that are associated with STAT3 activation are elevated in mucosal biopsies of

2009 Pickert et al. tion-Noncommercial-Share Alike-No Mirror Sites license for the first six months after the publication date (see http://www.jem.org/misc/terms.shtml). After six months it is available under a Creative Commons License (Attribution-Noncommercial-Share Alike 3.0 Unported license, as described at http://creativecommons .org/licenses/by-nc-sa/3.0/). 
IBD patients, and many of these cytokines have been shown to play an active role in the regulation of the inflammatory process (Atreya et al., 2000; Strober et al., 2007). It is therefore tempting to speculate that during the course of the inflammatory process, IECs respond to cytokines that may regulate the epithelial barrier via STAT3. In this report, we describe by using newly generated IEC-specific STAT3-deficient mice that STAT3 signaling in the intestinal epithelium is an important regulator of mucosal wound healing and is induced by IL-22 during acute experimental colitis.

\section{RESULTS AND DISCUSSION}

\section{Epithelial STAT3 activity regulates intestinal inflammation}

To study the regulation of intestinal epithelial STAT3 activity, we induced experimental colitis by treatment of mice with dextran sodium sulfate (DSS). Although at early time points (days 0 and 2) no significant epithelial STAT3 activity was detectable in the colon, STAT3 activity was present around day 5, as demonstrated by phosphorylated STAT3 (pSTAT3) staining of almost $80 \%$ of colonic IECs (Fig. 1 A). Colitis-associated induction of epithelial STAT3 activity was confirmed by Western blotting of epithelial cell lysates for pSTAT3 in mice with DSS-induced colitis (Fig. 1 B). To investigate the functional role of epithelial STAT3 acti- vation in experimental colitis, we generated mice with an IEC-specific deletion of STAT3 activity (STAT3 ${ }^{\text {IEC-KO). }}$ Accordingly, mice with floxed STAT3 alleles (Takeda et al., 1998) were crossbred to Villin-Cre mice expressing the Cre-recombinase specifically in IECs (Madison et al., 2002). Cre-mediated deletion of STAT3 was restricted to the colon and the small intestine of STAT3 $3^{\text {IEC-KO mice, whereas }}$ other organs remained unaffected (Fig. 1 C). Immunohistochemistry confirmed the specific absence of STAT3 phosphorylation in epithelial cells of STAT3 $3^{\mathrm{IEC}-\mathrm{KO}}$ mice (Fig. $1 \mathrm{D}$ ). STAT3 ${ }^{\mathrm{IEC}-\mathrm{KO}}$ mice developed normally and showed no spontaneous gut pathology upon histological analysis (Fig. $1 \mathrm{E}$ and Fig. S1). However, IECs of STAT3 ${ }^{\mathrm{IEC}-\mathrm{KO}}$ mice showed reduced expression of well-established target genes of the STAT3 signaling pathway, such as $b l_{X L}$ and socs3 (Fig. $1 \mathrm{~F}$ and Fig. S2 A; Levy and Darnell, 2002).

To investigate the functional role of epithelial STAT3 activation during colitis development, STAT3 ${ }^{\mathrm{IEC}-\mathrm{KO}}$ and control (STAT3fl) mice were challenged with 2.5\% DSS for $7 \mathrm{~d}$ to induce colitis. All mice developed colitis within $10 \mathrm{~d}$, as indicated by the time-dependent weight loss. Strikingly, however, although control mice were able to control weight loss after removing the DSS at day 7, STAT3 $3^{\text {IEC-KO mice lost }}$ significantly more weight after DSS treatment even after the
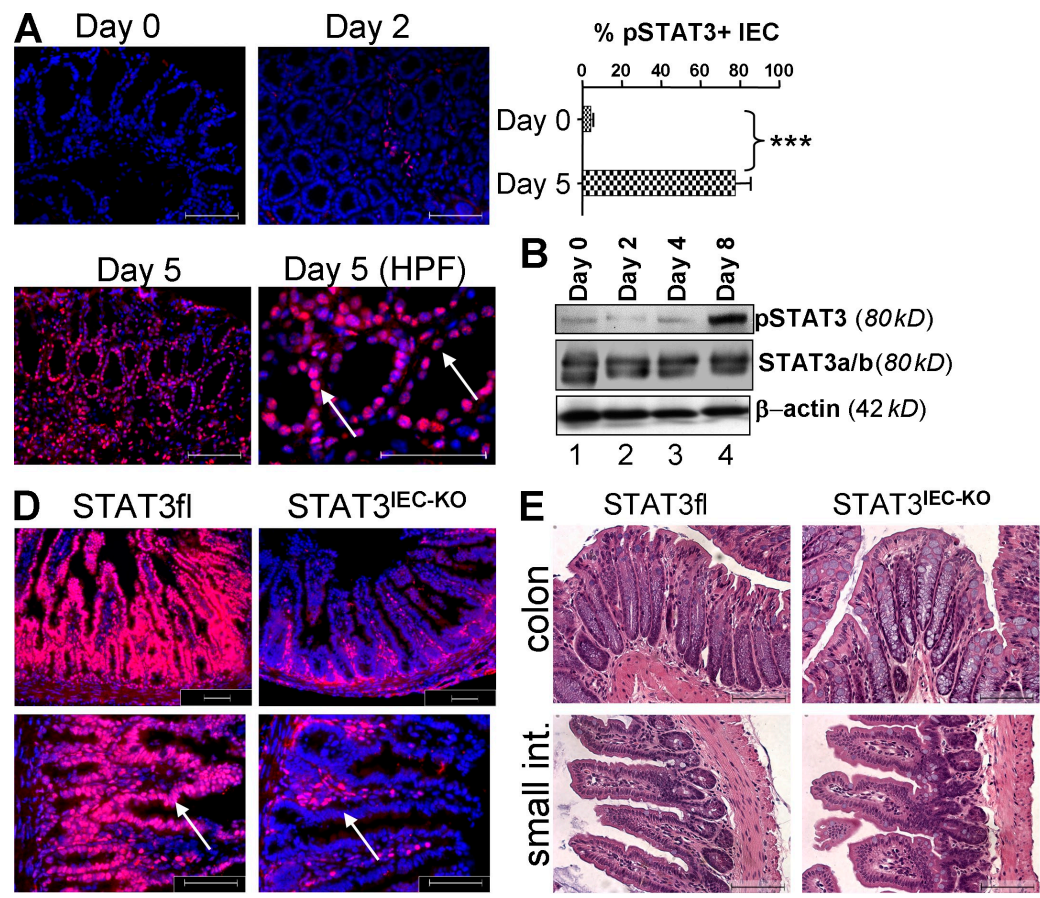

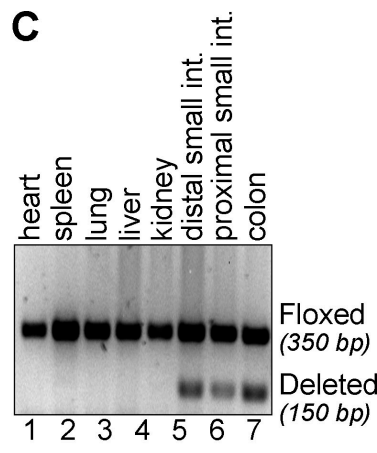

Figure 1. Generation and analysis of STAT3 ${ }^{\mathrm{IEC}-\mathrm{KO}}$ mice. (A) pSTAT3 staining of the colon from mice treated with DSS for up to 5 d. pSTAT3-positive IECS were counted from a total of 10 crypts at days 0 and 5 and are shown as the percentage of all counted IECs. Data show mean values \pm SD and are representative of three independent experiments. Arrows indicate the epithelial cell layer. ${ }^{* *}, P<0.001$. (B) Western blotting for pSTAT3 was performed from IECs isolated from wild-type mice at the indicated time points after DSS treatment. Probing of STAT3 and $\beta$-actin served as controls. The experiment was performed twice with similar results. (C) Presence of the deleted STAT3 allele selectively in the gut of STAT31EC-KO mice. DNA was isolated from a variety of organs and subjected to an allele-specific PCR. (D) Immunohistochemical analysis of pSTAT3 activity in the small intestine. Note the specific lack of pSTAT3 staining in IECS of STAT3 ${ }^{\mathrm{IEC}-\mathrm{KO}}$ mice (arrows). (E) H\&E staining of paraffin-embedded sections from 12-wk-old control and STAT3 ${ }^{\mathrm{IEC}-\mathrm{KO}}$ mice. (F) Changes in the expression levels of the STAT3 target genes $b \mathrm{c}_{X L}$ and socs3, as measured by qPCR from IECs isolated from the proximal (DD) and distal (II.) small intestine and the colon (Co.). Data show mean values of three mice per group \pm SD relative to HPRT. A second experiment gave similar results. Bars, $100 \mu \mathrm{m}$. HPF, high power field. 
DSS had been removed (Fig. 2 A). Methylene blue-aided endoscopy of the mice at the end of the experiment revealed more pronounced tissue damage with severe epithelial erosions, disruption of the normal vessel structure, and bleeding in STAT3 $3^{\mathrm{IEC}-\mathrm{KO}}$ mice when compared with control mice (Fig. 2, B and C; Becker et al., 2005). These findings were confirmed by histopathological analysis of colonic cross sections, in which we found significantly diminished numbers of colonic crypts and IECs in STAT3 ${ }^{\mathrm{IEC}-\mathrm{KO}}$ mice compared with controls (Figs. 2, D and E). To investigate whether the lack of epithelial STAT signaling may influence epithelial turnover, we analyzed proliferation and apoptosis in mice treated with DSS. Strikingly, STAT3 ${ }^{\mathrm{IEC}-\mathrm{KO}}$ mice showed less proliferation, as indicated by fewer KI-67+ ${ }^{+}$IECs per crypt when compared with control animals (Fig. 2 F). In addition, both active caspase- 3 and Tdt-mediated dUTP-biotin nick-end labeling (TUNEL) staining demonstrated a higher number of apoptotic IECs in STAT3 ${ }^{\mathrm{IEC}-\mathrm{KO}}$ mice (Fig. 2 G). Thus, in summary, our data indicate that epithelial STAT3 protects mice from colitis by regulating epithelial cell homeostasis.

\section{STAT3 during acute colitis regulates several pathways involved in mucosal wound healing}

To identify pathways potentially involved in the regulation of epithelial cell homeostasis by STAT3, we performed genome-wide expression analysis of control and STAT3 $3^{\mathrm{IEC}-\mathrm{KO}}$ epithelial cells of DSS-treated mice (available from the Gene Expression Omnibus under accession no. GSE15955). 52 out of the 45,000 examined expression tags were more than twofold down-regulated and 274 were up-regulated in epithelial cells from STAT3 ${ }^{\mathrm{IEC}-\mathrm{KO}}$ mice (Fig. 3 A). By gene ontology analysis, we found a significant enrichment of apoptosis-related genes in the gene cluster of the most up-regulated genes (Fig. 3 B, top). Conversely, a highly significant enrichment of genes associated with wound healing and the cellular stress response was observed in the gene cluster of the most repressed genes (Fig. 3 B, bottom). In an independent set of experiments, quantitative PCR studies were performed using isolated IECs from DSS-treated STAT3 $3^{\mathrm{IEC}-\mathrm{KO}}$ and control mice. Genes associated to apoptosis regulation ( $m c 11$ and survivin), proliferation (myc, Reg3b, and Pla2g5), and wound healing (smo) were significantly reduced in STAT3 ${ }^{\mathrm{IEC}-\mathrm{KO}}$ mice (Fig. S2 B). In summary, our data suggested that epithelial STAT3 orchestrates signaling pathways involved in IEC homeostasis and mucosal wound healing.

To investigate whether STAT3 promotes intestinal wound healing in the absence of DSS colitis, we performed woundhealing experiments in vivo. For this purpose, mucosal biopsies were taken from the colon of live mice using an

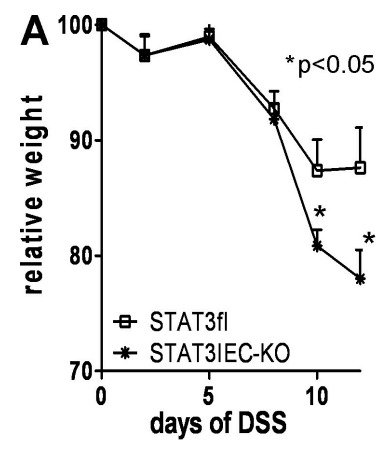

E

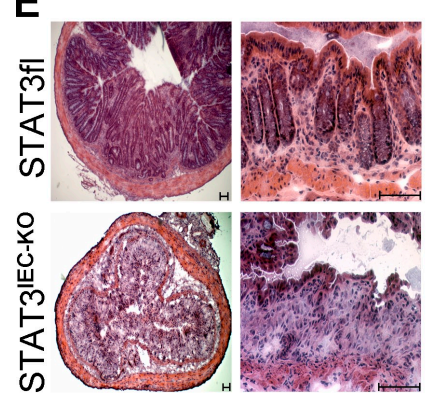

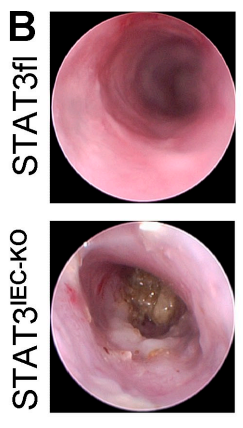

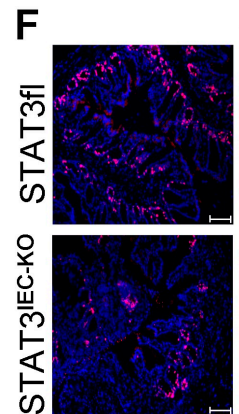

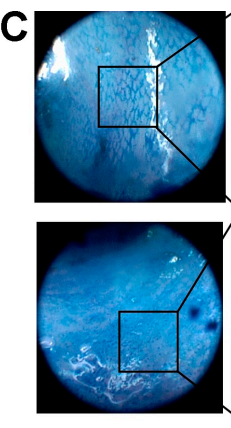
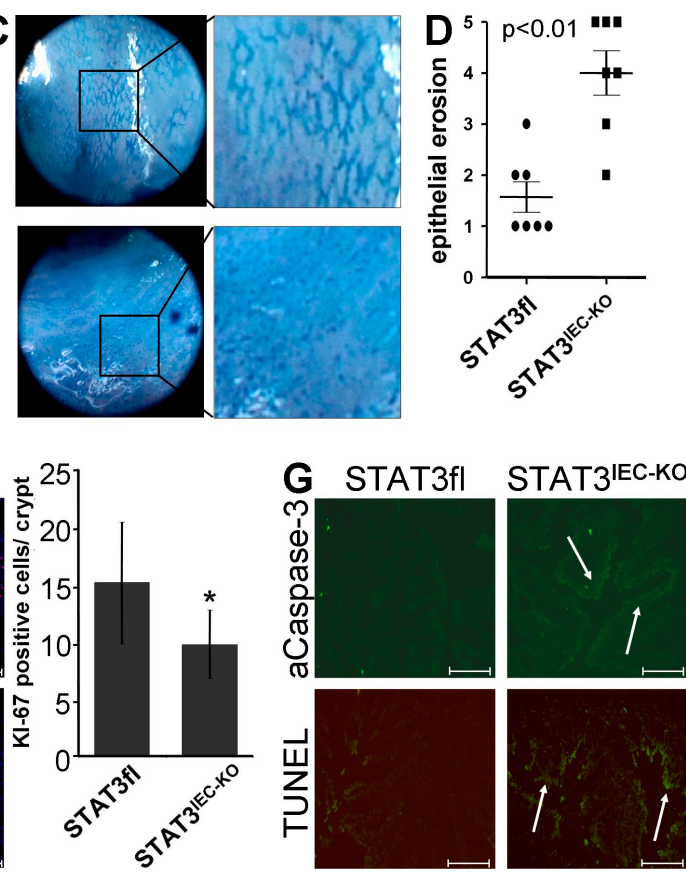

Figure 2. Severe colitis with epithelial erosions in STAT3 ${ }^{\mathrm{IEC}-\mathrm{Ko}}$ mice. (A) Control $(n=13)$ and STAT3 $3^{\mathrm{IEC}-\mathrm{KO}}(n=9)$ mice were treated with $2.5 \%$ DSS for 1 wk, as indicated in Materials and methods. Weight loss was monitored over time and is indicated as the percentage of the initial weight \pm SEM. The data are representative of four independent experiments. (B) Endoscopic examination of mice at day 15. (C) In vivo staining of the colon with methylene blue to visualize the colonic crypt pattern. (D) Histological analysis showing a severe loss of crypts. Epithelial erosion was scored as indicated in Materials and methods. Means of seven animals per group \pm SEM of one out of three independent experiments is shown. (E) Representative H\&E staining of the colon. (F) Immunostaining of the proliferation marker Ki-67 using colonic tissue samples of mice treated with DSS for 1 wk. Statistical analysis of Ki-67+ IECs per crypt $(n=8) .{ }^{*}, \mathrm{P}<0.05$. (G) Histochemical analysis of apoptosis using TUNEL and active caspase-3 staining. Representative images of four experiments are shown. Arrows indicate caspase or TUNEL+ epithelium. Bars, $100 \mu \mathrm{m}$. 
endoscopy-guided biopsy forceps, resulting in defined wounds of $\sim 800 \mu \mathrm{m}$ in size. The healing of these wounds was then monitored for several days, and the wound size was compared between control and STAT $3^{\mathrm{IEC}-\mathrm{KO}}$ mice by endoscopical analysis. Although in wild-type mice the wounds usually healed within $6 \mathrm{~d}$, wound healing was significantly delayed in STAT3 $3^{\mathrm{IEC}-\mathrm{KO}}$ mice and the wounds had still not closed at day 6 (Fig. 3 C). Furthermore, immunohistochemical analy- sis of the wound bed in wild-type mice $2 \mathrm{~d}$ after taking the biopsy indicated a localized induction of IEC STAT3 activity that was clearly confined to crypts adjacent to the wound bed (Fig. 3 D). Strikingly, wound-associated STAT3 activity colocalized with increased epithelial proliferation, as indicated by the presence of KI-67+ ${ }^{+}$IECs. Thus, our data support an important function of STAT3 signaling for mucosal wound healing.
A
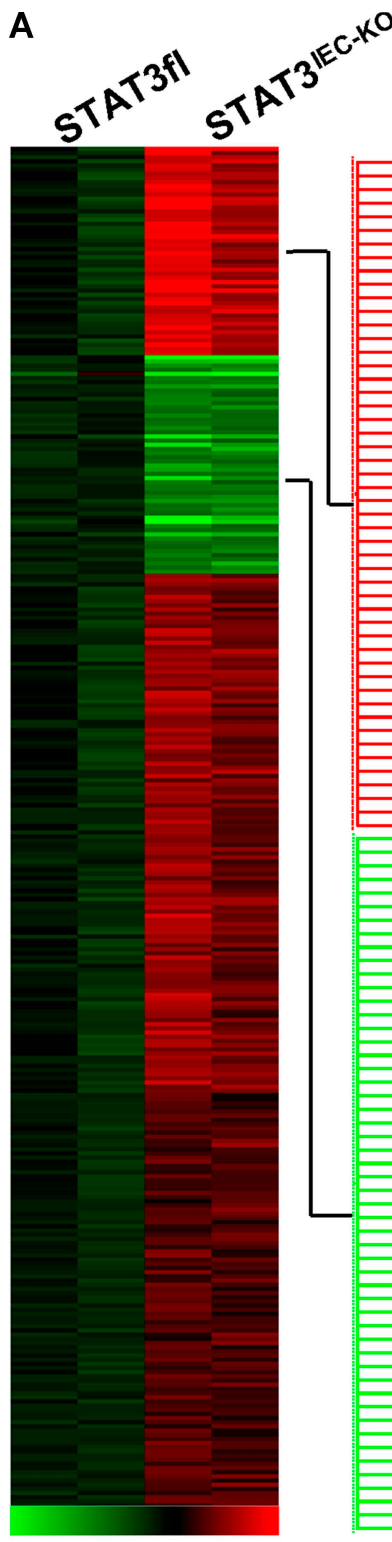

$-3$
B Down in STAT3 $3^{\mathrm{ECC}-K O}$ Reg3b Reg3 39 Lrg1
Trt
Socs 3 Socs3 Pla2g2a Fut9 Rla2g5 Slc35b2 Cpn2 Tac1 Slc5a9 Ptk6 Expi Casp14 Saa1. Gtf3c6 Ncald Saa2 Saa1 Hoxd13 101001 R $\begin{array}{ll}\text { Smo } & 2.18 \\ \text { 1110021J } 02 R i k & 2.17\end{array}$ $\begin{array}{ll}\text { Tmom64 } & 2.18 \\ \text { 1110021J02Rik } & 2.17\end{array}$

Up in STAT3IEC-KO STA
Mt1
Ppnr
Adar Adar Saps3 AW742560 Smad7 Aab130010C12Rik Zmiz1 Zmizi B830028B13Rik Ifi27 $\mathrm{Hrbl}$ AUt 015536 AU015536 Hsd3b2 Tmem164 5033418A18Rik Ldlr A930013F10Rik 2810403A07Rik C030013C21Rik 2610202C22Rik 3.95 $\begin{array}{ll}\text { 4921513D23Rik } & 3.94 \\ \text { Krt12 } & 3.93\end{array}$

\section{Metal ion binding Regulation of transcription Apoptosis} Regulation of cell cycle RNA metabolism Cell adhesion Cell differentiation

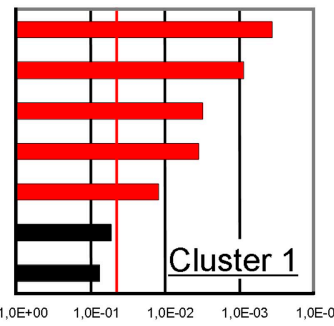

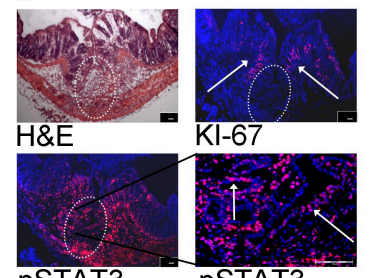

pSTAT3

C STAT3FI STAT3IEC-KO

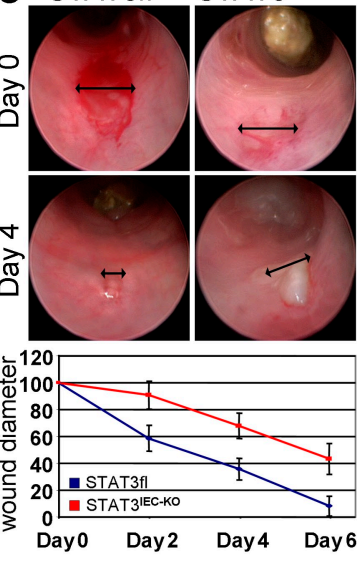

D

PSTAT3

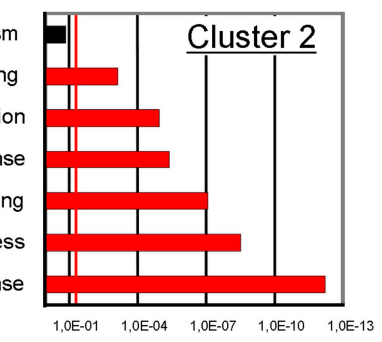

Figure 3. Intestinal epithelial STAT3 regulates mucosal wound healing. (A) Gene chip analysis of IECS from two control and two STAT3 ${ }^{1 E C-K O}$ mice. Mice were treated for $5 \mathrm{~d}$ with DSS, and IECs were isolated as described in Materials and methods. (B) Affymetrix expression data were analyzed using the gene ontology algorithm, as indicated in Materials and methods. The red line indicates $P<0.05$. Pathways reaching significance are shown in red. (C) In vivo wound-healing assay as described in Materials and methods. Wound healing was compared between control $(n=6)$ and STAT3IEC-KO $(n=5)$ mice by video endoscopies on days $0,2,4$ and 6 . Data show the mean diameters of the wound bed (double arrows) relative to the diameter of the fresh wound (percentage) of four to six wounds \pm SD. Representative images of three independent experiments are shown from days 0 and 4 . (D) Histochemical analysis of the wound bed (circle) $2 \mathrm{~d}$ after in vivo wounding in wild-type mice. pSTAT3 and $\mathrm{KI}-67^{+}$cells (arrows) were confined to crypts in close proximity to the wound. A second experiment gave similar result. Bars, $100 \mu \mathrm{m}$. 
STAT3 is specifically induced by IL-22 released from $\mathrm{CD}_{11} \mathrm{c}^{+}$cells during acute experimental colitis Depending on the target cells, STAT3 mediates signal transduction of several cytokines and proinflammatory mediators like IL-6 and IL-22 (Chapman et al., 2000; Levy and Darnell, 2002). Interestingly the latter two cytokines are strongly expressed in human patients with IBDs (Reinecker et al., 1993; Brand et al., 2006). Although both cytokines were undetectable in colonic biopsies at days 0 and 2 after DSS treatment, IL-22 was significantly induced at days 5 and 7, whereas IL-6 was detected at day 7 only (Fig. 4 A). Next, we compared epithelial STAT3 activity in DSS-treated wild-type, IL-6 ${ }^{-1-}$, and IL-22 $2^{-/-}$mice. Strikingly, although IL-6-deficient mice challenged with DSS for $1 \mathrm{wk}$ showed intestinal epithelial STAT3 activation indistinguishable from wild-type mice, IL-22 $2^{-/-}$mice showed an almost complete lack of epithelial STAT3 activity (Fig. 4 B). This finding was confirmed by Western blotting of IEC lysates from animals after $1 \mathrm{wk}$ of
DSS treatment showing a lack of pSTAT3 immunoreactivity in IECs from IL-22-deficient mice (Fig. 4 B). In agreement with previous reports (Zheng et al., 2008), we found that both IL-22 receptor chain genes (IL-22R and IL-10R2) were strongly expressed in the small and large bowel compared with other organs (Fig. S3). To provide evidence for a direct effect of IL-22 on IECs, full-thickness colon pieces from wild-type mice were cultured in the presence or absence of IL-22 and subsequently immunostained for pSTAT3. Although untreated colons did not show pSTAT3 immunoreactivity within the epithelium, treatment with IL-22 led to strong epithelial pSTAT3 staining (Fig. 4 C, top). This finding was further confirmed by Western blotting of primary IECs cultured in the presence of IL-22 (Fig. 4 C, bottom). Based on our observations, we propose that IL-22, via the activation of STAT3, protects the intestinal epithelium and promotes mucosal wound healing during acute experimental colitis. In support of this model, IL-22-/- mice developed
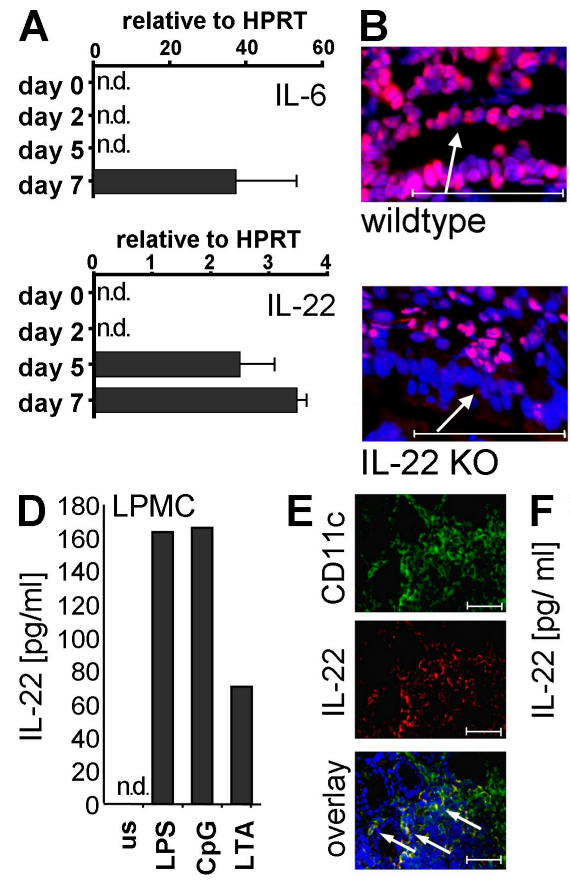
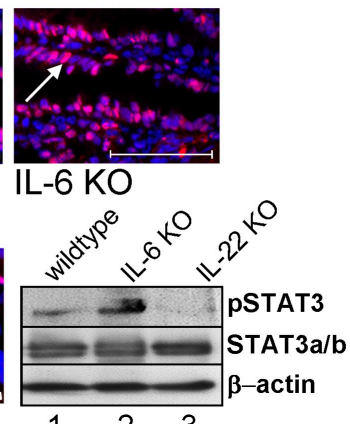
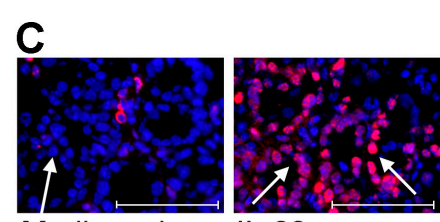

Medium alone IL-22

STAT3 ${ }^{\text {fl } / \text { fl }}$ STAT3 ${ }^{\text {IEC }}$-Ko

\begin{tabular}{llll|llll}
0 & 15 & 30 & 60 & 0 & 15 & 30 & 60 \\
\hline
\end{tabular}

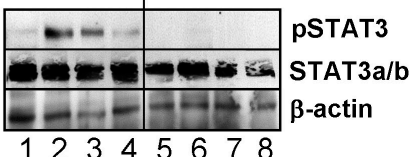

12345678
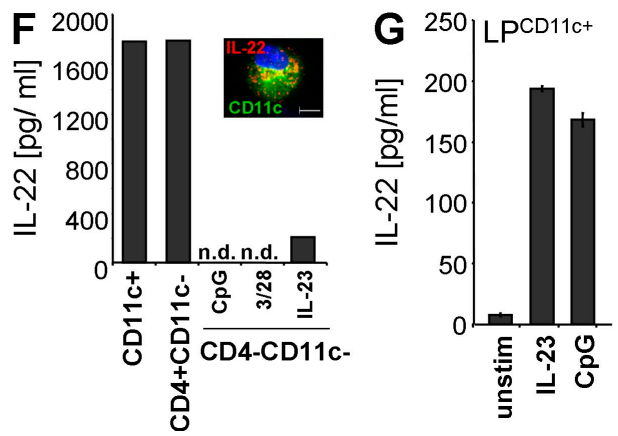

Figure 4. IL-22 directs epithelial STAT3 activity in acute DSS-induced colitis. (A) IL-6 and IL-22 expression ( \pm SD) in the gut of mice treated for 1 wk with DSS. Biopsies were taken by endoscopy at the indicated time points and analyzed by quantitative PCR. The experiment was performed twice with similar results. (B) Wild-type, IL-6 $6^{-1-}$, and IL-22-I- mice were treated with DSS for 1 wk. pSTAT3 immunostaining of the colonic epithelium (arrows). Representative images of three experiments are shown $(n=5)$. (bottom right) pSTAT3 Western blot using extracts of isolated IECs from this experiment. (C, top) pSTAT3 immunostaining of colon pieces isolated from wild-type mice and cultured in medium for $2 \mathrm{~h}$ in the presence or absence of recombinant IL-22. Representative images of three experiments with similar results are shown. Arrows indicate the epithelial cell layer. (bottom) pSTAT3 Western blot of IL-22-stimulated IECs isolated from control and STAT3IEC-KO mice. STAT3 and $\beta$-actin probing of the same blot served as a control. (D) LPMCs from DSS-treated wild-type mice were isolated as indicated in Materials and methods. Cells were stimulated in medium for $48 \mathrm{~h}$ as indicated, and supernatants were analyzed for IL-22 by ELISA. The experiment was performed three times with similar results. (E) Immunostaining for CD $11 \mathrm{c}$ and IL-22 of the inflamed colon of wild-type mice treated with DSS for 1 wk. The bottom image is an overlay of the single channels. Arrows indicate colocalization. Representative images of three experiments are shown. (F) IL-22 levels in purified lamina propria cells. LPMCs were isolated from five wild-type mice treated with DSS for $7 \mathrm{~d}$ and stimulated for $24 \mathrm{~h}$ as indicated. Supernatants were analyzed by ELISA. Data show cytokine expression of pooled cells from five mice of one experiment out of three. (inset) Colocalization of CD11c and IL-22 using immunostaining. (G) CD11 $\mathrm{c}^{+}$cells were isolated from the lamina propria of three wild-type mice and were stimulated as indicated. IL-22 was analyzed by ELISA from supernatants collected $24 \mathrm{~h}$ later. The experiment shows mean values \pm SD and was performed twice with similar results. Bars: (B, C, and E) $100 \mu$ m; (F) $10 \mu$ m. n.d., not detectable. 
more severe colitis than wild-type mice, and showed a loss of mucosal architecture and a lack of IECs comparable to that seen in STAT3 ${ }^{\mathrm{IEC}-\mathrm{KO}}$ mice (Fig. S4). Moreover, IL-22 ${ }^{-/-}$ mice showed delayed intestinal wound healing when compared with wild-type mice (Fig. S5). Similarly, DSS-induced colitis in wild-type mice that were treated with a neutralizing antibody against IL-22 was more severe than in controltreated animals (unpublished data). Therefore, our data suggest that IL-22 is an important regulator of mucosal wound healing via STAT3 activation in IECs.

To investigate the source of IL-22 in DSS-induced colitis, we first compared IL-22 expression and epithelial STAT3 activity in colon biopsies of DSS-treated wild-type and Rag1-/mice. Interestingly, we found that $\mathrm{Rag} 1^{-/-}$mice produced equal levels of IL-22 and induced epithelial STAT3 activity to a similar extent as compared with wild-type mice on day 7 after colitis induction (Fig. S6). We next isolated lamina propria cells from DSS-treated wild-type mice and stimulated them with LPS, CpG, and lipoteichoic acid. IL-22 was significantly induced by all three Toll-like receptor (TLR) ligands, therefore supporting a role for innate immune cell-derived IL-22 in the regulation of epithelial STAT3 activity (Fig. 4 D). To directly identify the source of IL-22 in the lamina propria of mice with colitis, we performed immunostaining of colon cross sections from animals with established DSS colitis for CD11c and IL-22. As demonstrated in Fig. 4 E, CD11c cells were enriched in the inflamed colon. Moreover, we observed a striking colocalization of CD11c and IL-22, suggesting that DCs may be an important source for IL-22 in the inflamed colon. To further confirm these findings, we sequentially purified $\mathrm{CD} 11 \mathrm{c}^{+}, \mathrm{CD}^{+}$, and $\mathrm{CD} 11 \mathrm{c}^{-} \mathrm{CD} 4^{-}$cells from the lamina propria of inflamed colon tissues and cultured them for 24 h. IL-22 was present in both $\mathrm{CD} 11^{+}$and $\mathrm{CD} 4^{+}$cell cultures, but only little IL-22 was produced by $\mathrm{CD} 4^{-} \mathrm{CD} 11 \mathrm{c}^{-}$cells (Fig. 4 F). Furthermore, when isolated cells were immunostained for CD11c and IL-22, we observed colocalization of these markers (Fig. 4 F, inset). To investigate whether CD11 $\mathrm{c}^{+}$cell expression of IL-22 was limited to colitis, we isolated CD11 $\mathrm{c}^{+}$ cells from the lamina propria of healthy mice and stimulated them with $\mathrm{CpG}$ and IL-23, which was recently described as an inducer of IL-22 in DCs (Zheng et al., 2008). Again, we found that IL-22 expression was markedly induced in lamina propria CD11c cells (Fig. $4 \mathrm{G}$ ), suggesting that DCs stimulated by TLR ligands and IL-23 are an important source of IL-22 in acute colitis.

In summary, our observations provide evidence that epithelial STAT3 functions as an important regulator of tissue homeostasis in the gut. STAT3 is induced in IECs after induction of colitis or injury, and it regulates several signaling pathways that are commonly involved in wound healing. Our study furthermore provides evidence that STAT3 activation during acute colitis is critically dependent on IL-22. IL-22 has been described to be produced by activated T cells, especially by Th17 cells as well as by DCs and NK cells (Wolk and Sabat, 2006; Ouyang et al., 2008). Furthermore, in a very recent report, a distinct $\mathrm{NK}$ cell population $\left(\mathrm{NKp} 46^{+}\right)$ was described as an innate source of IL-22 in human and mouse lymphoid tissue (Cella et al., 2009). In this report, we confirm that IL-22 during acute colitis is produced by cells of the innate immune system, as well as by $\mathrm{CD} 4^{+} \mathrm{T}$ cells. However, $\mathrm{CD}^{+} \mathrm{T}$ cells are present only in small numbers in the lamina propria during the initial acute phase of DSS-induced colitis and are largely confined to lymphoid follicles (unpublished data). In contrast, CD $11 c^{+}$DCs are present in significant numbers in the colon of healthy animals and are strategically located directly underneath the surface epithelium (Rimoldi et al., 2005). Thus, activation of these DCs via TLRs either by invading pathogens or by transepithelial sampling of bacteria may promote rapid activation of the IL-22STAT3 axis at the intestinal epithelium. Our model is in keeping with a recent study showing that Myd88-mediated signaling through mesenchymal cells is required for the amplification of mouse colonic epithelial progenitors that occurs in response to DSS-induced injury (Pull et al., 2005). However, our data do not exclude that in chronic colitis or at later stages of established colitis, IL-22 may also be significantly expressed by other inflammatory cells infiltrating the lamina propria, such as Th17 cells, or that other factors may be expressed that may also stimulate IEC STAT3 activity. Among those lines, NK cells have recently been established as producers of IL-22 and some NK cell populations express CD11c (Zenewicz et al., 2008). Although our data confirm that cells expressing the NK cell marker DX5 can produce IL-22, little IL-22 was found in supernatants of $\mathrm{DX} 5^{+}$cells isolated from the lamina propria of mice treated with DSS (Fig. S7).

Our model is in agreement with previous reports suggesting that IL-22 is a central regulator of tissues in inflammatory conditions, and several studies have previously suggested a protective role for IL-22 in the regulation of gut inflammation. Accordingly, IL-22 gene delivery was shown to ameliorate DSS-induced colitis (Sugimoto et al., 2008). In another study, in a model of experimental Citrobacter rodentium infection, IL-22 $2^{-/-}$mice showed increased intestinal epithelial damage, along with systemic bacterial burden and significantly increased mortality, when compared with control mice (Zheng et al., 2008). In this study, IL-22 induced the expression of Reg proteins, which are released by IECs and exert antimicrobial functions. This observation is in keeping with our present observation that STAT3 ${ }^{\mathrm{IEC}-\mathrm{KO}}$ mice showed greatly diminished expression of RegIII $\gamma$ and RegIII $\beta$ in gene chip experiments (Fig. 3), suggesting that STAT3 functions as a key regulator for the expression of these genes. IL-22-induced STAT3 activation in the injured gut may therefore not only promote IEC proliferation and protection against apoptosis but may also serve to control bacterial growth in the lamina propria adjacent to the wound. Our data are also consistent with two very recently published studies in which IL-6/gp130mediated STAT3 activation during tumor development was shown to drive cell proliferation of enterocytes (Bollrath et al., 2009; Grivennikov et al., 2009). In conclusion, IL-22/ STAT3 signaling in IECs emerges as an important protective pathway for the mucosal immune system. STAT3 seems 
dispensable for gut homeostasis under steady-state conditions but is activated upon challenge to drive tissue regeneration and protection in situations of increased demand, as during colitis and injury.

\section{MATERIALS AND METHODS}

Mice. Mice with a floxed STAT3 allele were provided by S. Akira (Osaka University, Osaka, Japan; Takeda et al., 1998). Villin-Cre mice were received from D. Gumucio and B. Madison (University of Michigan Medical School, Ann Arbor, MI) and were described earlier (Madison et al., 2002). IL-6 ${ }^{-1-}$ mice were purchased from the Jackson Laboratory. IL-22 $2^{-/-}$mice generated by Lexicon Pharmaceuticals and Genentech have been previously described (Zheng et al., 2007). Mice with an IEC-specific deletion of STAT3 activity (STAT3 ${ }^{\mathrm{IEC}-\mathrm{KO}}$ ) were generated by breeding floxed STAT3 mice with VillinCre mice. Wild-type C57BL/6 and RAG1 ${ }^{-1-}$ mice were obtained from the animal facility of the University of Mainz. All mice were kept under specific pathogen-free conditions. Animal protocols were approved by the Institutional Animal Care and Use Committee of the University of Mainz.

Western blotting. Western blotting was performed as previously described (Becker et al., 2004). Anti-STAT3, pSTAT3 (Cell Signaling Technology), or $\beta$-actin (Santa Cruz Biotechnology, Inc.) antibodies and the ECL Western blotting analysis system (GE Healthcare) were used.

Experimental mouse models and mouse endoscopy. Colitis was induced by giving mice 2.5\% DSS (MP Biomedicals) in the drinking water for $1 \mathrm{wk}$. DSS was exchanged every other day. For monitoring of colitis, the Coloview high resolution mouse endoscopic system (Karl-Storz) was used (Becker et al., 2005).

In vivo wound-healing experiments. For the analysis of wound healing in vivo, mucosal lesions with a diameter of $800 \mu \mathrm{m}$ were generated in healthy mice using biopsy forceps introduced through the working channel of the endoscope. The healing process was monitored by endoscopic examination every other day. The diameter of the wound was calculated relative to the original wound size by video analysis of endoscopical recordings.

Isolation and culture of intestinal cells. Mouse lamina propria mononuclear cells (LPMCs) and IECs were isolated as previously described (Becker et al., 2004). LPMCs were collected from the interface of a 40:80 Percoll gradient. For some experiments, $\mathrm{CD} 11 \mathrm{c}^{+}, \mathrm{CD}^{+}$, or $\mathrm{DX} 5^{+}$cells were further purified from LPMCs using immunomagnetic beads directed against CD11c (Miltenyi Biotec).

Cytokine quantification. Cells were cultured for $48 \mathrm{~h}$ in medium (X-Vivo 15; Lonza) in the presence or absence of the following stimuli: $1 \mu \mathrm{g} / \mathrm{ml} \mathrm{LPS}$ (InvivoGen), $1 \mu \mathrm{M} \mathrm{CpG} \mathrm{(Eurofins} \mathrm{MWG),} \mathrm{lipoteichoic} \mathrm{acid} \mathrm{(Merck),}$ $10 \mu \mathrm{g} / \mathrm{ml}$ anti-mouse CD3e (clone 145-2C11) and $5 \mu \mathrm{g} / \mathrm{ml}$ anti-mouse CD28 (clone 37.51), or $50 \mathrm{ng} / \mathrm{ml} \mathrm{IL-23} \mathrm{(R \& D} \mathrm{Systems).} \mathrm{Full-thickness} \mathrm{co-}$ lon pieces $\left(3 \mathrm{~mm}^{2}\right)$ were cultured in X-Vivo 15 medium for $48 \mathrm{~h}$. Supernatants were collected and subjected to IL-22 ELISA according to the manufacturer's protocol (Bender MedSystems).

Gene expression analysis. Total RNA was isolated using the RNA micro kit (QIAGEN). Generation of cDNA was achieved using Stratascript II RT (Invitrogen). Real-time quantitative PCR was performed on the iQ iCycler (Bio-Rad Laboratories) with cDNA-specific Quantitect Primer assays (QIAGEN) and Absolute QPCR SYBR Green Fluorescein Mix (Abgene). Gene chip experiments were performed by the Mainz University core facility using the mouse 4302.0 chip (Affymetrix). For multiple gene array testings, including differential expression analysis and clustering, the software package ArrayAssist (Agilent Technologies) was used, and the elimination of redundancies in gene lists and subsequent gene ontology-based gene class testings were performed with Pathway architect software (Agilent Technologies).
Histochemistry. Histopathological analysis was performed on formalin-fixed tissue after hematoxylin and eosin (H\&E) staining. Epithelial erosion was scored as loss of crypts: $1,0-20 \% ; 2,20-40 \% ; 3,40-60 \% ; 4,60-80 \%$; and 5 , $80-100 \%$. Immunofluorescence of cryosections was performed using the TSA Cy3 system (PerkinElmer) and a fluorescence microscope (IX70; Olympus; Becker et al., 2004) using primary antibodies directed against pSTAT3, Ki-67 (Dako), and active caspase-3 (Cell Signaling Technology) and CD11c (BD). The nuclei were counterstained with Hoechst 3342 (Invitrogen). Apoptosis (TUNEL) was analyzed using the in situ cell death detection kit (Roche).

Statistical analysis. Data were analyzed by the Student's $t$ test using Excel (Microsoft).

Online supplemental material. Fig. S1 shows that STAT3 ${ }^{\text {IEC-KO }}$ mice have no obvious gut phenotype, and Fig. S2 demonstrates changes in the expression levels of STAT3 target genes in unchallenged and DSS-treated control and STAT3 ${ }^{\mathrm{IEC}-\mathrm{KO}}$ mice. Fig. S3 demonstrates expression of IL-22 receptor chains in IECs. Figs. S4 and S5 show DSS colitis and in vivo wound healing in IL-22 $2^{-1-}$ mice. Fig. S6 demonstrates IL-22/STAT3 signaling in $\mathrm{RAG1}^{-/-}$mice. Fig. S7 shows IL-22 expression in isolated lamina propria $\mathrm{CD} 11 \mathrm{c}^{+}$cells. Online supplemental material is available at http://www.jem .org/cgi/content/full/jem.20082683/DC1.

We thank A. Nikolaev, H. Zepp, and G. Carlino for excellent technical assistance. The research of C. Becker and M.F. Neurath was supported by the Collaborative Research Centers SFB548 and SFB432 of the Deutsche Forschungsgemeinschaft (DFG). G. Pickert was supported by a grant from the state of Rhineland-Palatinate. M. Leppkes and S. Hirth were supported by the graduate school GK 1043/2 of the DFG.

W. Ouyang is an employee of Genentech and Y. Zheng is an employee of Pfizer Global Research and Development. All other authors declare no conflicting financial interests.

Submitted: 26 November 2008

Accepted: 15 June 2009

\section{REFERENCES}

Akira, S. 2000. Roles of STAT3 defined by tissue-specific gene targeting. Oncogene. 19:2607-2611.

Alonzi, T., I.P. Newton, P.J. Bryce, E. Di Carlo, G. Lattanzio, M. Tripodi, P. Musiani, and V. Poli. 2004. Induced somatic inactivation of STAT3 in mice triggers the development of a fulminant form of enterocolitis. Cytokine. 26:45-56.

Atreya, R., J. Mudter, S. Finotto, J. Mullberg, T. Jostock, S. Wirtz, M. Schutz, B. Bartsch, M. Holtmann, C. Becker, et al. 2000. Blockade of interleukin 6 trans signaling suppresses T-cell resistance against apoptosis in chronic intestinal inflammation: Evidence in Crohn disease and experimental colitis in vivo. Nat. Med. 6:583-588.

Becker, C., M.C. Fantini, C. Schramm, H.A. Lehr, S. Wirtz, A. Nikolaev, J. Burg, S. Strand, R. Kiesslich, S. Huber, et al. 2004. TGF-beta suppresses tumor progression in colon cancer by inhibition of IL-6 trans-signaling. Immunity. 21:491-501.

Becker, C., M.C. Fantini, S. Wirtz, A. Nikolaev, R. Kiesslich, H.A. Lehr, P.R Galle, and M.F. Neurath. 2005. In vivo imaging of colitis and colon cancer development in mice using high resolution chromoendoscopy. Gut. 54:950-954.

Bollrath, J., T.J. Phesse, V.A. von Burstin, T. Putoczki, M. Bennecke, T. Bateman, T. Nebelsiek, T. Lundgren-May, O. Canli, S. Schwitalla, et al. 2009. gp130-mediated Stat3 activation in enterocytes regulates cell survival and cell-cycle progression during colitis-associated tumorigenesis. Cancer Cell. 15:91-102.

Brand, S., F. Beigel, T. Olszak, K. Zitzmann, S.T. Eichhorst, J.M. Otte, H. Diepolder, A. Marquardt, W. Jagla, A. Popp, et al. 2006. IL-22 is increased in active Crohn's disease and promotes proinflammatory gene expression and intestinal epithelial cell migration. Am. J. Physiol. Gastrointest Liver Physiol. 290:G827-G838.

Cella, M., A. Fuchs, W. Vermi, F. Facchetti, K. Otero, J.K. Lennerz, J.M Doherty, J.C. Mills, and M. Colonna. 2009. A human natural killer cell 
subset provides an innate source of IL-22 for mucosal immunity. Nature. 457:722-725.

Chapman, R.S., P. Lourenco, E. Tonner, D. Flint, S. Selbert, K. Takeda, S. Akira, A.R. Clarke, and C.J. Watson. 2000. The role of Stat3 in apoptosis and mammary gland involution. Conditional deletion of Stat3. Adv. Exp. Med. Biol. 480:129-138.

Grivennikov, S., E. Karin, J. Terzic, D. Mucida, G.Y. Yu, S. Vallabhapurapu, J. Scheller, S. Rose-John, H. Cheroutre, L. Eckmann, and M. Karin. 2009. IL-6 and Stat3 are required for survival of intestinal epithelial cells and development of colitis-associated cancer. Cancer Cell. 15:103-113.

Levy, D.E., and J.E. Darnell Jr. 2002. Stats: transcriptional control and biological impact. Nat. Rev. Mol. Cell Biol. 3:651-662.

Liu, X., Y.S. Lee, C.R. Yu, and C.E. Egwuagu. 2008. Loss of STAT3 in CD4+ $\mathrm{T}$ cells prevents development of experimental autoimmune diseases. J. Immunol. 180:6070-6076.

Lovato, P., C. Brender, J. Agnholt, J. Kelsen, K. Kaltoft, A. Svejgaard, K.W. Eriksen, A. Woetmann, and N. Odum. 2003. Constitutive STAT3 activation in intestinal T cells from patients with Crohn's disease. J. Biol. Chem. 278:16777-16781.

Madison, B.B., L. Dunbar, X.T. Qiao, K. Braunstein, E. Braunstein, and D.L. Gumucio. 2002. Cis elements of the villin gene control expression in restricted domains of the vertical (crypt) and horizontal (duodenum, cecum) axes of the intestine. J. Biol. Chem. 277:33275-33283.

Mudter, J., B. Weigmann, B. Bartsch, R. Kiesslich, D. Strand, P.R. Galle, H.A. Lehr, J. Schmidt, and M.F. Neurath. 2005. Activation pattern of signal transducers and activators of transcription (STAT) factors in inflammatory bowel diseases. Am. J. Gastroenterol. 100:64-72.

Nenci, A., C. Becker, A. Wullaert, R. Gareus, G. van Loo, S. Danese, M. Huth, A. Nikolaev, C. Neufert, B. Madison, et al. 2007. Epithelial NEMO links innate immunity to chronic intestinal inflammation. Nature. 446:557-561.

Ouyang, W., J.K. Kolls, and Y. Zheng. 2008. The biological functions of T helper 17 cell effector cytokines in inflammation. Immunity. 28:454-467.

Pull, S.L., J.M. Doherty, J.C. Mills, J.I. Gordon, and T.S. Stappenbeck. 2005. Activated macrophages are an adaptive element of the colonic epithelial progenitor niche necessary for regenerative responses to injury. Proc. Natl. Acad. Sci. USA. 102:99-104.

Reinecker, H.C., M. Steffen, T. Witthoeft, I. Pflueger, S. Schreiber, R.P. MacDermott, and A. Raedler. 1993. Enhanced secretion of tumour necrosis factor-alpha, IL-6, and IL-1 beta by isolated lamina propria mononuclear cells from patients with ulcerative colitis and Crohn's disease. Clin. Exp. Immunol. 94:174-181.

Rescigno, M. 2008. The pathogenic role of intestinal flora in IBD and colon cancer. Curr. Drug Targets. 9:395-403.

Rimoldi, M., M. Chieppa, V. Salucci, F. Avogadri, A. Sonzogni, G.M. Sampietro, A. Nespoli, G. Viale, P. Allavena, and M. Rescigno. 2005. Intestinal immune homeostasis is regulated by the crosstalk between epithelial cells and dendritic cells. Nat. Immunol. 6:507-514.

Strober, W., I. Fuss, and P. Mannon. 2007. The fundamental basis of inflammatory bowel disease. J. Clin. Invest. 117:514-521.

Sugimoto, K., A. Ogawa, E. Mizoguchi, Y. Shimomura, A. Andoh, A.K. Bhan, R.S. Blumberg, R.J. Xavier, and A. Mizoguchi. 2008. IL-22 ameliorates intestinal inflammation in a mouse model of ulcerative colitis. J. Clin. Invest. 118:534-544

Takeda, K., T. Kaisho, N. Yoshida, J. Takeda, T. Kishimoto, and S. Akira. 1998. Stat3 activation is responsible for IL-6-dependent T cell proliferation through preventing apoptosis: generation and characterization of $\mathrm{T}$ cellspecific Stat3-deficient mice. J. Immunol. 161:4652-4660.

Takeda, K., B.E. Clausen, T. Kaisho, T. Tsujimura, N. Terada, I. Forster, and S. Akira. 1999. Enhanced Th1 activity and development of chronic enterocolitis in mice devoid of Stat3 in macrophages and neutrophils. Immunity. 10:39-49.

Welte, T., S.S. Zhang, T. Wang, Z. Zhang, D.G. Hesslein, Z. Yin, A. Kano, Y. Iwamoto, E. Li, J.E. Craft, et al. 2003. STAT3 deletion during hematopoiesis causes Crohn's disease-like pathogenesis and lethality: a critical role of STAT3 in innate immunity. Proc. Natl. Acad. Sci. USA. 100:1879-1884.

Wolk, K., and R. Sabat. 2006. Interleukin-22: a novel T- and NK-cell derived cytokine that regulates the biology of tissue cells. Cytokine Growth Factor Rev. 17:367-380.

Zenewicz, L.A., G.D. Yancopoulos, D.M. Valenzuela, A.J. Murphy, S. Stevens, and R.A. Flavell. 2008. Innate and adaptive interleukin-22 protects mice from inflammatory bowel disease. Immunity. 29:947-957.

Zheng, Y., D.M. Danilenko, P. Valdez, I. Kasman, J. Eastham-Anderson, J. $\mathrm{Wu}$, and W. Ouyang. 2007. Interleukin-22, a T(H)17 cytokine, mediates IL-23-induced dermal inflammation and acanthosis. Nature. 445:648-651.

Zheng, Y., P.A. Valdez, D.M. Danilenko, Y. Hu, S.M. Sa, Q. Gong, A.R. Abbas, Z. Modrusan, N. Ghilardi, F.J. de Sauvage, and W. Ouyang. 2008. Interleukin-22 mediates early host defense against attaching and effacing bacterial pathogens. Nat. Med. 14:282-289. 